\title{
Increased sphincter of Oddi basal pressure in patients affected by gall stone disease: a role for biliary stasis and colicky pain?
}

\author{
M Cicala, F I Habib, F Fiocca, N Pallotta, E Corazziari
}

\begin{abstract}
Background and aims-Even if the motor activity of the gall bladder and sphincter of Oddi (SO) are integrated, it is not known if the presence of stones in the gall bladder affects SO function. The aim of the study was to compare SO motor activity in patients with and without gall stones.

Patients and methods-In a series of 155 patients consecutively submitted to endoscopic retrograde cholangiopancreatography and SO manometry for suspected biliary or pancreatic disease, 23 gall stone patients had recurrent episodes of biliary or pancreatic pain (colicky group); 52 patients had non-biliary/pancreatic-type abdominal pain/discomfort, and of these, 15 had gall stones (non-colicky group), 25 were free of stones (controls), and 12 had undergone cholecystectomy.

Results-SO basal pressure in gall stone patients in the colicky or non-colicky group was significantly higher than in controls $(p<0.001)$. SO basal pressure recorded in postcholecystectomy patients did not differ from controls. SO phasic activity did not differ between the patient groups. SO dysfunction was detected in more than $40 \%$ of gall stone patients irrespective of associated biliary/pancreatic pain but in none of the control subjects $(\mathrm{p}<0.001)$.
\end{abstract}

Conclusions-Gall stones are frequently associated with increased SO tone which may obstruct bile flow thus acting to facilitate gall bladder stasis, and may play a role as a cofactor in biliary/pancreatic pain.

(Gut 2001;48:414-417)

Keywords: biliary pain; gall bladder; gall stone disease; sphincter of Oddi

To better understand the mechanism of stone formation and origin of colicky pain in gall stone patients, many investigations have focused on modifications in gall bladder motility. ${ }^{1-9}$ However, their role in these conditions is still far from clear. Thus although several studies, both in animals ${ }^{10-14}$ and humans, ${ }^{15-19}$ have provided evidence supporting the role of gall bladder bile stasis in the genesis of gall stones, clear cut data on impaired gall bladder contractility have not been found consistently in gall stone patients.

Although colicky pain in gall stone patients is generally thought to be caused by the increased pressure within a gall bladder contracting against a cystic duct obstructed by a stone(s), identical biliary-type pain occurs in postcholecystectomy patients, in patients with a choledochal stone(s), and in those without stones and sphincter of Oddi (SO) dysfunction. ${ }^{20}$ Furthermore, it has been reported that in patients without $^{21}$ and with gall stones, ${ }^{22}$ biliary colic may be caused by SO dysfunction and increased pressure within the common bile duct rather than by gall bladder contractility and increased pressure within the gall bladder.

Taking into account the fact that the motor activity of the gall bladder and $\mathrm{SO}$ are integrated and linked by neural reflexes, ${ }^{23-27}$ it may be hypothesised that dysfunction of the SO may be present in patients with a stone(s) in the gall bladder. Hence the aim of the present study was to evaluate SO motor activity and to assess the occurrence of SO dysfunction in patients with gall stones.

\section{Methods}

PATIENTS

The study population comprised 155 consecutive patients with recurrent upper abdominal pain and/or discomfort referred for endoscopic retrograde cholangiopancreatography (ERCP) and SO manometry as part of a diagnostic workup for suspected biliary or pancreatic disease.

Gall bladder status was assessed by means of an oral cholecystogram and/or real time ultrasonography. Sixty one patients with concomitant disorders of the liver, pancreas, or biliary tract (with the exception of gall bladder stones) were excluded from the study. Nineteen patients $(20 \%)$ were excluded from the analysis for technical reasons (10 for failure of cannulation and nine for artefacts on the manometric tracings).

The first group (colicky group) comprised 23 patients (16 females, mean age 51.3 (SD 14) years, range 14-85) who had gall stones and complied with the Rome diagnostic criteria for biliary or pancreatic pain ${ }^{28}$ : moderate to severe steady pain located in the epigastrium and/or right upper abdominal quadrant, lasting 20 minutes, which has occurred on one or more occasions during the past 12 months, sometimes accompanied by biochemical features of transient biliary and/or pancreatic tract obstruction (elevated serum transaminase and/or alkaline phosphatase and/or conjugated

Abbreviations used in this paper: ERCP, endoscopic retrograde cholangiopancreatography; LFTs, liver function tests; SO, sphincter of Oddi. 
bilirubin (LFTs) and/or amylase and/or lipase). In this group, 14 patients had elevation of one or more LFTs and/or amylase/lipase during episodes of pain.

The remaining 52 patients had upper abdominal pain not in keeping with the Rome diagnostic criteria of biliary or pancreatic pain in terms of type and duration. None presented with increased LFTs or amylase/lipase and bile ducts and pancreatic duct were normal at ERCP. Of these patients, 15 (eight females, mean age 48.6 (18) years, range 26-80) had gall stones (non-colicky group), 25 (16 females, mean age 49.2 (15) years, range 23-74) were free of stones (control group), and 12 (seven females, mean age 46.0 (13) years, range 31-60) had undergone cholecystectomy for uncomplicated gall stone disease 3-6 years previously (cholecystectomy group).

Informed consent was obtained from all patients prior to the investigation, and the study protocol was approved by the local ethics committee.

\section{METHODS}

Immediately before endoscopy, patients were sedated with diazepam (10-20 mg intravenously). After completion of ERCP, ${ }^{29}$ manometry was performed using a $200 \mathrm{~cm}$ polyethylene triple lumen catheter with three distal side holes located $2 \mathrm{~mm}$ apart (Arndorfer Medical Specialties, Inc, Greendale, Wisconsin, USA), continuously infused with bubble free water at a rate of $0.25 \mathrm{ml} / \mathrm{min}$ by a minimally compliant hydraulic capillary infusion system (Arndorfer). The catheter, passed through the biopsy channel of a duodenoscope (Olympus Co., TJF 100/TJF 140, Tokjo, Japan), was introduced into the common bile duct and withdrawn across the $\mathrm{SO}$ in $2 \mathrm{~mm}$ step increments. The correct position was confirmed at fluoroscopy during injection of small amounts of contrast medium through the catheter. The catheter was then positioned to record SO motor activity for at least two minutes with all three manometric sensors; intraduodenal pressures were recorded by a single lumen catheter taped to the endoscope. Pressure recordings were divided into and measured over one minute periods. SO maximal basal pressure was measured at the mid inspiratory phase and expressed as $\mathrm{mm} \mathrm{Hg}$, with duodenal pressure as a zero reference. Mean SO basal pressure represented the mean of all maximal basal pressures, recorded at a steady baseline of at least $20 \mathrm{sec}-$ onds. Maximal basal pressure was the highest

Table 1 Mean (SD) values for sphincter of Oddi (SO) resting pressures and phasic activity variables in the four groups of patients

\begin{tabular}{|c|c|c|c|c|}
\hline & $\begin{array}{l}\text { Gall stone } \\
\text { colicky } \\
(n=23)\end{array}$ & $\begin{array}{l}\text { Gall stone } \\
\text { non-colicky } \\
(n=15)\end{array}$ & $\begin{array}{l}\text { Controls } \\
(n=25)\end{array}$ & $\begin{array}{l}\text { Cholecystectomy } \\
(n=12)\end{array}$ \\
\hline \multicolumn{5}{|l|}{ Resting SO pressure } \\
\hline $\begin{array}{l}\text { Mean SO basal pressure }(\mathrm{mm} \mathrm{Hg}) \\
\text { Maximal SO basal pressure }(\mathrm{mm}\end{array}$ & $23.1(14)^{\star}$ & $18.9(8)$ & $14.3(6)$ & $14.7(6)$ \\
\hline $\mathrm{Hg})$ & $47.2(30)^{\star \star}$ & $36.2(17)^{\star \star}$ & $21.5(8)$ & $26.2(10)$ \\
\hline \multicolumn{5}{|l|}{ Phasic activity } \\
\hline Frequency (cycle/min) & $2.5(1.3)$ & $1.7(1.9)$ & $3.1(1.4)$ & $2.2(0.7)$ \\
\hline Duration (s) & $6(1.6)$ & $5.7(1.6)$ & $6.1(1.6)$ & $5.9(1.6)$ \\
\hline Amplitude (mm Hg) & $91(24)$ & $94(37)$ & $101(31)$ & $70(16)$ \\
\hline
\end{tabular}

${ }^{\star} \mathrm{p}<0.02 ;{ }^{\star \star} \mathrm{p}<0.001 v$ controls. value recorded. The amplitude of SO contractions was measured from the peak to the base of the waves and expressed as $\mathrm{mm} \mathrm{Hg}$ with $\mathrm{SO}$ maximal basal pressure as the zero reference. Duration of SO phasic contractions was measured from the onset of the ascending to the end of the descending tract of the wave. Frequency of SO contractions was measured as the number of waves per minute. Each investigated variable was assessed at each recording level and expressed as the average of the mean values of the three tracings.

SO mean and maximal basal pressure, as well as amplitude, duration, and frequency of SO phasic contractions were evaluated in the four groups. A maximal $\mathrm{SO}$ basal pressure equal to or exceeding $40 \mathrm{~mm} \mathrm{Hg}$ was considered as evidence of SO dysfunction. ${ }^{30}$

STATISTICAL ANALYSIS

The Mann-Whitney U test and Fisher's test were used to assess intergroup differences.

\section{Results}

Mean and maximal basal pressures of the SO recorded in gall stone patients with biliary or pancreatic pain (colicky group) were significantly higher (23.1 (14) mm Hg and 47.2 (30) $\mathrm{mm} \mathrm{Hg}$, respectively) compared with those in patients with intact gall bladder and no stones complaining of pain not apparently suggestive of a biliary or pancreatic source (control group) (respectively, 14.3 (6) $\mathrm{mm} \mathrm{Hg}, \mathrm{p}<0.02$; and 21.5 (8) $\mathrm{mm} \mathrm{Hg}, \mathrm{p}<0.001$ ). Of the gall stone patients without typical biliary or pancreatic pain and not presenting abnormal LFTs, amylase or lipase (non-colicky group), SO maximal basal pressure was significantly higher than in the control group (36.2 (17); $\mathrm{p}<0.001)$.

Mean and maximal basal pressures (14.7 (6); 26.2 (10) $\mathrm{mm} \mathrm{Hg}$ ) recorded in subjects who previously underwent cholecystectomy (cholecystectomy group) did not differ statistically from controls (table 1, fig 1).

Phasic activity of the SO, in terms of duration, frequency, and amplitude of contractions, did not differ significantly between the two groups of gall stone patients (colicky and non-colicky groups); also, there was no significant difference between the cholecystectomy group and controls (table 1 ).

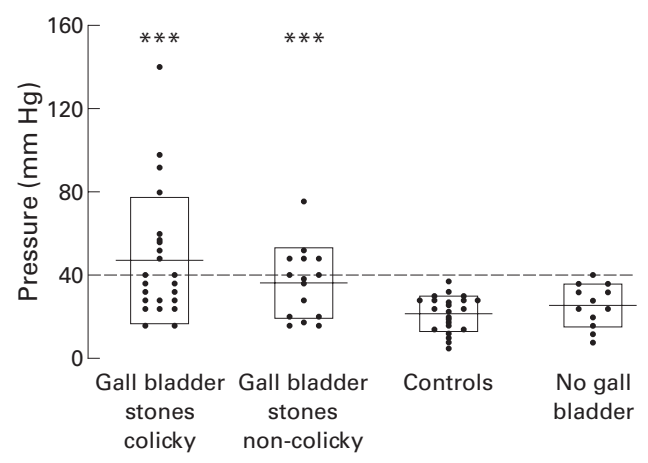

Figure 1 Distribution of individual data for sphincter of Oddi maximal basal pressures recorded in the four groups of patients. Broken line represents the cut off normal value of $40 \mathrm{~mm} \mathrm{Hg} ;{ }^{\star \star *} p<0.001 v$ the control group. 
SO dysfunction (maximal SO pressure $\geqslant 40$ $\mathrm{mm} \mathrm{Hg}$ ) was detected in none of the patients in the control group, in 11 of 23 (48\%) in the colicky group $(p<0.001)$, in seven of $15(46 \%)$ in the non-colicky group $(\mathrm{p}<0.001)$, and in one of 12 in the cholecystectomy group ( $8 \%$, NS $v$ controls).

Complications following ERCP and SO manometry included pancreatitis in six patients $(6.4 \%)$ : one severe, one moderate, and four mild.

\section{Discussion}

Although it has been indicated that the gall bladder and SO can reciprocally affect motor function, ${ }^{23-27}$ little attention has focused on the motor activity of the SO in gall stone patients and it is not known if the presence of stones in the gall bladder affects the gall bladder-SO interrelationship.

Three studies have reported data on SO pressure in gall stone patients. The first was limited to analysis of SO phasic pressure which was comparable in a mixed group of gall stone and cholecystectomised patients and did not offer any indications concerning SO basal pressure. ${ }^{31}$ In the second, ${ }^{32}$ SO motor activity was recorded in a small series of gall stone patients and the average value of mean SO basal pressure was higher than that in postcholecystectomy patients. In the third, ${ }^{33}$ which assessed SO motor activity in patients with recurrent pancreatitis, $\mathrm{SO}$ basal pressure in the control group of asymptomatic gall stone patients was not different from that in normal controls, and thus at variance with our findings reported here. A more recent investigation by those same authors, ${ }^{34}$ however, on SO motor function in patients with symptomatic gall stones and a normal common bile duct revealed that $4 \%$ of patients with normal alkaline phosphatase and $40 \%$ of comparable patients with increased alkaline phosphatase presented an abnormally elevated SO basal pressure.

In the present study, to evaluate the association, if any, of gall stone disease with impaired SO motor activity, SO manometry was performed in patients with and without gall stones. The results showed that none of the latter group had elevated SO maximal and mean basal pressure whereas a substantial number of the former group had elevated SO pressures.

As colicky pain in gall stone patients may originate in the gall bladder and also in the SO as a result of the possible concomitant presence of SO fibrosis and/or migration of stones from the gall bladder to the duodenum, gall stone patients not complaining of biliary/pancreatictype pain and with no laboratory evidence of abnormal LFTs or pancreatic enzymes were evaluated and the results compared with those from gall stone patients undergoing a diagnostic workup for recurrent biliary/pancreatic-type pain and with those from patients without gall stones.

Exclusion of several patients from the analysis for technical reasons may have had a limited effect on the relative difference between groups but the finding that all gall stone patients, irrespective of clinical presentation and laboratory findings, had similar maximal and mean SO basal pressures which were significantly more elevated than in those without gall stones, would indicate that the presence of stones in the gall bladder is frequently associated with elevated SO pressure.

The finding of normal SO pressure in patients without gall stones and in those who previously underwent cholecystectomy for biliary colic and gall stones suggests that elevated SO basal pressure in gall stone patients is secondary to the presence of the stones in the gall bladder.

Increased SO basal pressure may not be devoid of marked effects on bile flow regulation. For example, it is known that maximal basal pressure is the SO motor variable which best correlates with hepatoduodenal bile transit time, at least in postcholecystectomy subjects ${ }^{25} 35$; in addition, several investigations in animals and humans indicate that SO sphincterotomy is followed by a reduction in gall bladder volume and improved gall bladder emptying function, even in the presence of gall stones. ${ }^{1{ }^{14}}{ }^{36-41}$ It can therefore be hypothesised that the increased SO pressure in gall stone patients would inhibit bile flow into the duodenum and facilitate bile flow into, and storage in, the gall bladder thus offering a possible explanation for the common finding of an increase in fasting gall bladder volume in gall stone patients. $^{7-9}$

An unexpected result in the present study was the finding of SO maximal basal pressure equal to or exceeding $40 \mathrm{~mm} \mathrm{Hg}$ in at least $46 \%$ of all gall stone patients, irrespective of whether or not they presented with episodes of biliary or pancreatic-type pain and evidence of abnormal LFTs. A possible explanation could be selection bias as the study population was comprised of patients referred for upper abdominal pain. It cannot be ruled out that modifications in SO pressure may play a role in the pathogenesis of both colicky and noncolicky pain. This selection bias could have been further reinforced by the small number of patients in each group.

The manometric evidence in the postcholecystectomy patients of maximal SO basal pressure equal to or exceeding $40 \mathrm{~mm} \mathrm{Hg}$ and no residual stones as well as complaints of biliary/ pancreatic-type pain is diagnostic of SO dysfunction which is usually considered an indication for therapeutic sphincterotomy in this group of symptomatic patients. ${ }^{20}$

Within the limits of the present study it is impossible to establish if the finding of an elevated SO basal pressure in patients with a gall bladder in situ and containing stones but with no evidence of biliary/pancreatic-type pain and/or altered liver or pancreatic function tests is clinically significant. Elevated SO basal pressure in gall stone patients may be considered an important predisposing pathogenetic cofactor which, in the presence of other transient conditions such as the passage of crystals and/or SO spasm and/or inflammation, may give rise to biliopancreatic symptoms. 
Increased SO basal pressure may possibly create a condition which would predispose to colicky pain resulting in the need for cholecystectomy in non-colicky patients and would result in postcholecystectomy pain in those patients who underwent cholecystectomy for biliopancreatic manifestations.

A longitudinal study on colicky gall stone patients before and after cholecystectomy as well as in dyspeptic non-colicky gall stone patients and in healthy controls is warranted to confirm the present hypotheses and to offer an insight into the potential pathogenetic implications of increased SO basal pressure in gall stone patients.

The authors are grateful to Mrs Marian Shields for revision of the English language.

1 Fisher RS, Stelzer F, Rock E, et al. Abnormal gallbladder emptying in patients with gallstones. Dig Dis Sci 1982;27:1019-24.

2 Forgacs IC, Maisey MN, Murphy GM, et al. Influence of gallstones and ursodeoxycholic acid therapy on gallbladder emptying. Gastroenterology 1984;87:299-307.

3 Masclee AAM, Jansen JBMJ, Driessen WWM, et al. Plasma cholecystokinin and gallbladder response to intraduodenal fat in gallstone patients. Dig Dis Sci 1989;34:353-9.

4 Pomeranz IS, Shaffer EA. Abnormal gallbladder emptying in a subgroup of patients with gallstones. Gastroenterology in a subgroup of

5 Maugdal DP, Kupfer RM, Zentler-Munro PL, et al. Postprandial gallbladder emptying in patients with gallPostprandial gallbladder emp

6 Portincasa P, Stolk MF, van Erpecum KJ, et al. Cholesterol gallstone formation in man and potential treatments of the gallbladder motility defect. Scand $\mathcal{F}$ Gastroenterol 1995; (suppl) 212:63-78

7 Festi D, Frabboni R, Bazzoli F, et al. Gallbladder motility in cholesterol gallstone disease. Effect of ursodeoxycholic acid administration and gallstone dissolution. Gastroenterology 1990;99:1779-85.

8 Van Erpecum KJ, van Berge Henegouwen GP, Stolk MFJ, et al. Fasting gallbladder volume, postprandial emptying and cholecystokinin release in gallstone patients and norma subjects. F Hepatol 1992;14:194-202.

9 Pauletzki J, Cicala M, Holl J, et al. Correlation between gallbladder fasting volume and postprandial emptying in patients with gallstones and healthy controls. Gut 1993;34: patients

10 Lee SP, LaMont JT, Carey MC. Role of gallbladder mucus hypersecretion in the evolution of cholesterol gallstones. Studies in the prairie dog. 7 Clin Invest 1981;67:1712-23.

11 Lee SP, Carey MC, LaMont JT. Aspirin prevention of cholesterol gallstone formation in prairie dogs. Science 1981; 211:1429-31

12 Roslyn JJ, DenBesten L, Thompson JE, et al. Chronic cholelithiasis and decreased bile salt pool size. Cause or effect? Am $\mathcal{F}$ Surg 1980;139:119-24

13 Hutton SW, Sievert CE Jr, Vennes JA, et al. The effect of sphincterotomy on gallstone formation in the prairie dog. Gastroenterology 1981;81:663-7.

14 Hutton SW, Sievert CE Jr, Vennes JA, et al. Inhibition of gallstone formation by sphincterotomy in the prairie dog:

Roslyn JJ, Pitt HA, Man LL

et al. Gallbladder disease in patients on longterm parenteral nutrition. Gastroenterology $1983 ; 84: 148-54$

16 Everson GT, McKinley C, Lawson M, et al. Gallbladder function in human females: effect of the ovulatory cycles, pregnancy and contraceptive steroids. Gastroenterology pregnancy and

17 Van Liessum PA, Hopman WPM, Peeters GF, et al. Postprandial gallbladder motility during long term treat- ment with the long-acting somatostatin analog SMS 201-995 in acromegaly. 7 Clin Endocrinol Metab 1989;69: 557-62.

18 Ahrendt SA, McGuire GE, Pitt HA, et al. Why does somatostatin cause gallstones? Am $\mathcal{F}$ Surg 1991;161:177-82.

19 Fisher RS, Rock E, Levin G, et al. Effects of somatostatin on gallbladder emptying. Gastroenterology 1987;92:885-90.

20 Geenen JE, Hogan WJ, Dodds WJ, et al. The efficacy of endoscopic sphincterotomy after cholecystectomy in patients with sphincter of Oddi dysfunction. $N$ Engl 7 Med 1988;320:82-7.

21 Ruffolo TA, Sherman S, Lehman GA, et al. Gallbladder ejection fraction and its relationship to sphincter of Oddi dysfunction. Dig Dis Sci 1994;39:289-92.

22 Basso N, Bagarani M, Materia A, et al. Effects of cerulein in patients with biliary colic pain. Gastroenterology 1985;89: 605-9.

23 Padbury RTA, Furness JB, Kuramato H, el al. Direct neural projections from the duodenum to the gallbladder and sphincter of Oddi. Gastroenterology 1990;98:A379.

24 Thune A, Jivengard L, Conradi N, et al. Cholecystectomy in the cat damages pericholedochal nerves and impairs reflex regulation of the sphincter of Oddi. Acta Chir Scand Suppl 1988;154:191-4.

25 Thune A, Saccone GTP, Scicchitano JP, et al. Distension of the gallbladder inhibits sphincter of Oddi motility in man. Gut 1991;32:690-3.

26 Thune A, Thornell E, Svanvik J. Reflex regulation of flow resistance in the feline sphincter of Oddi by hydrostatic pressure in the biliary tree. Gastroenterology 1986;91:13649.

27 Simula ME, Meedeniya ACB, Toouli J, et al. Projection and neurochemical coding of circular muscle motor neurons innervating the sphincter of Oddi ( $\mathrm{SO}$ ) in the Australian opossum. Neurogastroenterol Motil 1999;11:291.

28 Corazziari E, Shaffer EA, Hogan WJ, et al. Functional disorders of the biliary tract and pancreas. Gut 1999;45(suppl 2):II $48-54$

29 Dodds WJ. Biliary tract motility and its relationship to clinical disorders. AfR Am f Roentgenol 1990;155:247-58.

30 Corazziari E, Cicala M, Habib FI, et al. Hepatoduodenal bile transit in cholecystectomized subjects. Relationship with sphincter of Oddi function and diagnostic value. Dig Dis Sci 1994;39:1985-93.

31 Csendes A, Kruse A, Funch-Jensen P, et al. Pressure measurements in the biliary and pancreatic duct systems in controls and in patients with gallstones, previous cholecystectomy, or common bile duct stones. Gastroenterology 1979;77:1203-10

32 Torsoli A, Biliotti D, Habib FI, et al. Common bile duct stones and sphincter of Oddi function. Ital $\mathcal{F}$ Gastroenterol 1986;18:109-11.

33 Guelrud M, Mendoza S, Vicent S, et al. Pressures in the sphincter of Oddi in patients with gallstones, common duct stones and recurrent pancreatitis. F Clin Gastroenterol 1983; 5:37-41.

34 Guelrud M, Mendoza S, Mujica V, et al. Sphincter of Oddi (SO) motor function in patients with symptomatic gallstones. Gastroenterology 1993;104:A361.

35 Cicala M, Scopinaro F, Corazziari E, et al. Quantitative cholescintigraphy in the assessment of choledochoduodenal bile flow. Gastroenterology 1991;100:1106-13.

36 Hutton SW, Sievert CE, Vennes JA, et al. Spontaneous passage of glass beads from the canine gallbladder: facilitation by sphincterotomy. Gastroenterology 1988;94:1031-5.

$37 \mathrm{Li}$ YF, Weisbrodt NW, Moody FG. Effect of bile diversion and sphincterotomy on gallbladder muscle contractility and gallstone formation. Am $\mathcal{F}$ Surg 1991;162:31-5.

38 Cohn MS, Schwartz SI, Faloon WW, et al. Effect of sphincteroplasty on gallbladder function and bile composition. Ann Surg 1979;189:317-21.

39 Tanaka M, Ikeda S, Yoshimoto H, et al. The longterm fate of the gallbladder after endoscopic sphincterolomy: complete follow-up study of 122 patients. Am f Surg 1987;154:5059.

40 Sugiyama M, Atomi Y. Longterm effects of endoscopic sphincterotomy on gallbladder motility. Gut 1996;39:8569 .

41 Sharma BC, Agarwal DK, Baijal SS, et al. Effect of endoscopic sphincterotomy on gall bladder bile lithogenicity and motility. Gut 1998;42:288-92. 